\title{
A SPECTRAL METHOD FOR SOLVING ELLIPTIC EQUATIONS FOR SURFACE RECONSTRUCTION AND 3D ACTIVE CONTOURS
}

\author{
Jia Li and Alfred O. Hero \\ Department of Electrical Engineering and Computer Science \\ University of Michigan \\ 1301 Beal Avenue, Ann Arbor, MI 48105 USA
}

\begin{abstract}
The solution of elliptic partial differential equations arises in 3D surface reconstruction and active contours. Most current approaches are iterative including finite element methods (FEM) and finite difference methods (FDM). In this paper, we describe a fast spectral method for solving elliptic equations over the unit sphere. A double Fourier series expansion is applied to model convex or starshaped 3D surfaces. The Helmholtz equation governing a diffusion on the unit sphere is solved by spectral methods using double Fourier series as orthogonal basis functions. The optimization of the regularization parameter, which controls the tradeoff between denoising and matching high spatial frequencies, is studied for different 3D shapes and noise models. We show how the resultant solution can be combined with active contour methods to speed up 3D medical image segmentation. A number of examples and simulation results are presented to illustrate the algorithm.
\end{abstract}

\section{INTRODUCTION}

The need to solve elliptic equations of the general form

$$
\nabla^{2} u-\epsilon u=f
$$

arises in several computer vision problem, such as shape from shading [1], surface reconstruction [2] and active contours [3]. These problems can be formulated in the framework of variational principles and lead to solving Euler-Lagrange equations of elliptic type as the necessary condition for a minimum. Although there exist direct analytical methods for solving these equations on 2D rectangular domain [4], current approaches that we are aware of solve these equations defined over closed 3D surface by iterative techniques [3]. In this paper, we apply spectral methods [5, 6] to solve elliptic equations over the unit sphere and apply this to the problems of surface reconstruction and 3D active contours.

A 3D surface is usually defined by a mapping $\mathrm{x}: \Omega \rightarrow \Re^{3}$, i.e. $\mathbf{x}(v, w)=\left(x_{1}(v, w), x_{2}(v, w), x_{3}(v, w)\right)$, where $\Omega$ is a $2 \mathrm{D}$ domain and $(v, w) \in \Omega$. In this paper, we will only consider closed surfaces which are star-shaped. With the assumption that the origin has been aligned up with the object center, these surfaces can be represented by a radial descriptor $r(\theta, \phi): S^{2} \rightarrow \Re$ defined over the unit sphere $S^{2}$ in $\Re^{3}$. Throughout this paper, the following notations are used:

$I(x, y, z), 3 \mathrm{D}$ grey-level image;

This work was partly supported by the National Cancer Institute, United States Department of Health and Human Services under Grant R01 CA87955. $\mathbf{x}(v, w)$, surface function in Cartesian coordinates; $u(\theta, \phi)$, admissible surface function in spherical coordinates; $f(\theta, \phi)$, segmentation data in surface reconstruction problem or distance between the origin and the nearest edge point of $u(\theta, \phi)$ in $3 \mathrm{D}$ active contour problem;

$d(x, y, z)$, Euclidean distance from a point $(x, y, z)$ on the $3 \mathrm{D}$ active contour to its nearest edge point;

$\epsilon$ and $\alpha$, parameters controlling tradeoff.

\section{PDE'S IN RECONSTRUCTION AND SEGMENTATION}

There are many computer vision problems which can be formulated as optimization via variational principles. These variational principles lead to solving one or more elliptic PDE's. Some of these problems are: shape from shading, height from gradient, surface reconstruction and optical flow. Below we discuss the formulation of surface reconstruction and 3D active contours in the context of variational principles.

\subsection{Surface Reconstruction}

The goal of surface reconstruction is to find a credible surface that can smooth noisy surface sample data provided by a coarse segmentation algorithm. Assuming the origin has been aligned with an interior point of the object, we use $f(\theta, \phi)$ to represent the segmentation data specified on the unit sphere and $u(\theta, \phi)$ to represent the admissible surface function which satisfy smoothness constraint and data fidelity constraint. A common formulation of this problem is to define an energy functional $E$ which is a sum of the energy contributions from the smoothness constraint and the data constraint,

$$
E(u)=\int_{S^{2}} Z(u) d \Omega+\epsilon \int_{S^{2}} Y(u, f) d \Omega,
$$

where $Z$ is a roughness measure which encapsulates the irregularity of the surface $u, Y$ measures the discrepancy between the surface $u$ and the segmentation data $f$, and $\epsilon$ is a regularization parameter controlling the tradeoff between denoising and matching high spatial frequencies in the segmentation data. The smoothness term should penalize the gradient of the function $u$ to enforce smoothness. For instance, $Z$ can be defined as $Z(u)=\|\nabla u\|^{2}$, where $\nabla$ is the gradient operator. A common definition of the data fidelity metric is $Y(u, f)=(u-f)^{2}$. With these choices, the energy functional takes the form:

$$
E(u)=\int_{S^{2}}\|\nabla u\|^{2} d \Omega+\epsilon \int_{S^{2}}(u-f)^{2} d \Omega
$$


The final solution to the reconstruction problem should be a critical point $u=u^{*}$ which minimizes the energy functional $E(u)$. The solution must therefore satisfy the following Euler-Lagrange equation on the unit sphere [7]:

$$
\nabla^{2} u-\epsilon(u-f)=0 .
$$

This is a special case of the Helmholtz equation on the unit sphere. The solution to this equation will be discussed in Section 3 .

\subsection{D Active Contours}

In the last two decades, active contour methods have been developed to solve the image segmentation problem and the reconstruction problem simultaneously. The approaches can be classified into parametric active contours and geometric active contours. A typical 3D parametric active contour deforms under internal forces and external forces, ultimately taking the shape of the object boundary which minimizes the contour's associated energy functional $E(\mathbf{x})$ :

$$
E(\mathbf{x})=\int_{\Omega}\left[\alpha\|\nabla \mathbf{x}\|^{2}+\beta\left\|\nabla^{2} \mathbf{x}\right\|^{2}\right] d \Omega+\int_{\Omega} P_{e x t}(I, \mathbf{x}) d \Omega
$$

where $\mathbf{x}$ denotes the contour in Cartesian coordinates and $I$ is the 3D image. The energy functional $E$ is composed of the internal energy, the first integral term which is computed from the contour itself, and the external energy, the second integral term which is computed from both the contour and the image data. The force generated by the internal energy discourages stretching and bending of the contour having the effect of regularization on the contour. On the other hand, the force generated by the external energy attracts the contour towards the boundary.

Increasing $\alpha$ increases the resistance to stretching force, so that the contour tends to shrink toward a solution that reduces the active contour curve length or surface area. On the other hand, increasing $\beta$ increases the resistance to tensile stress and bending. In this paper, $\beta$ is set to zero to allow second-order discontinuity in the contour.

The external energy is usually defined to be a potential function which has a global minimum at the object boundary. Typical potential functions designed to deform the active contour are $P_{(1)}=h_{1}(\nabla I), P_{(2)}=h_{2}(d)$, where $d$ is defined in the Section $1, h_{1}$ and $h_{2}$ are convex potential functions. Potential functions of type $P_{(1)}$ have the disadvantage that the resulting external force has very small capture range because the value of $P_{(1)}$ is near zero in regions where the intensity is nearly homogeneous. Potential functions of type $P_{(2)}$ solve this problem by incorporating edge points extracted by local edge detectors. The locations of edge points are broadcasted to many of their neighbors. When $P_{e x t}$ is designed to equal $d^{2}$, the energy functional becomes

$$
E(\mathbf{x})=\int_{\Omega} \alpha\|\nabla \mathbf{x}\|^{2} d \Omega+\int_{\Omega} d^{2} d \Omega
$$

Let us replace $\mathbf{x}$, the surface representation in cartesian coordinates by $u(\theta, \phi)$, the surface representation in spherical coordinates and replace the distance potential $d^{2}$ by $(u-f)^{2}$, where $f(\theta, \phi)$ denotes the distance between the origin and the nearest edge point of $u(\theta, \phi)$. The equation (6) becomes

$$
E(u)=\int_{S^{2}} \alpha\|\nabla u\|^{2} d \Omega+\int_{S^{2}}(u-f)^{2} d \Omega
$$

which is analogous to equation (3) both in expression and in physical interpretation. The Euler-Lagrange equation must have the same form as (4). Other types of external potential functions may generate Poisson type Euler-Lagrange equations, which can also be solved by the spectral method described in Section 3 .

In the case of surface reconstruction, where the elliptic equation only needs to be solved once, it is often possible to apply FEM or FDM to obtain a solution in a reasonable amount of time. When a 3D active contour is in evolution, $f$ is updated in every iteration, therefore the elliptic equation has to be solved many times before the contour converges to the object boundary. In this case, the iterative method must be applied for each update which is often too slow for practical applications. This was verified by Cohen, who used finite element methods to solve the PDE in [3].

\section{THE SPECTRAL METHOD}

Spectral methods for solving PDE's over a 2D rectangular domain are well known for their faster rate of convergence and higher accuracy as compared to iterative FEM and FDM methods. These methods usually take advantage of symmetries by transforming the equation into spectral domain and only require $O\left(N^{2} \log N\right)$ operations for a 2D problem on $N \times N$ grid. It was Simchony who first applied spectral method to solve Poisson equations in computer vision problems [4]. Although similar methods for solving PDE over unit sphere have been used in numerical weather prediction and the study of ocean dynamics $[5,6]$, to the best of our knowledge, they have not been used in computer vision. In the subsequent sections, we discuss the application of spectral methods to solve elliptic equations 4 for surface reconstruction and evolving $3 \mathrm{D}$ active contours.

\subsection{Double Fourier Series Expansion}

If we move the term $\epsilon f$ in the equation (4) to the right hand side and define it as the force function $f$ in the Helmholtz equation (1), (4) can be rewritten as $\nabla^{2} u-\epsilon u=f$, or more explicitly:

$$
\frac{1}{\sin \theta} \frac{\partial}{\partial \theta}\left(\sin \theta \frac{\partial u}{\partial \theta}\right)+\frac{1}{\sin ^{2} \theta} \frac{\partial^{2} u}{\partial^{2} \phi}=\epsilon u+f
$$

where $\theta \in[0, \pi]$ is the polar angle and $\phi \in[0,2 \pi]$ is the azimuthal angle in spherical coordinates. As in many problems with sphere geometry [8], we expand the scalar function $u$, and similarly for $f$, with a Fourier series in longitude with a truncation $M$,

$$
u(\theta, \phi)=\sum_{m=-M}^{M} u_{m}(\theta) e^{i m \phi},
$$

where $u_{m}(\theta)=\frac{1}{K} \sum_{k=0}^{K-1} u\left(\theta, \phi_{k}\right) e^{-i m \phi_{k}}, \phi_{k}=2 \pi k / K$ and $K=2 M$. Substituting this expansion into equation (8), we obtain the ODE

$$
\frac{1}{\sin \theta} \frac{d}{d \theta} \sin \theta \frac{d}{d \theta} u_{m}(\theta)-\frac{m^{2}}{\sin ^{2} \theta} u_{m}(\theta)=\epsilon u_{m}(\theta)+f_{m}(\theta)
$$

The next key step is to approximate the function $u_{m}(\theta)$, and similarly for $f_{m}(\theta)$, with truncated sine or cosine functions,

$$
u_{m}(\theta)=\sum_{n=0}^{J-1} u_{n, m}\left[\left(1-s_{m}\right) \cos n \theta+s_{m} \sin n \theta\right]
$$


where $s_{m}=0$ for even $m$ and $s_{m}=1$ for odd $m$. This enables us to reduce the equation (10) to an algebraic system of equations in the Fourier space:

$$
\begin{gathered}
{[(n-1)(n-2)+\epsilon] u_{n-2, m}-2\left[\left(n^{2}+2 m^{2}\right)+\epsilon\right] u_{n, m}} \\
+[(n+1)(n+2)+\epsilon] u_{n+2, m} \\
=-f_{n-2, m}+2 f_{n, m}-f_{n+2, m}
\end{gathered}
$$

For a given $m$, the components of even and odd $n$ are uncoupled. Therefore the original equation (10) is composed of two independent subsystems. We can write them in matrix form:

$$
D \mathbf{u}=A \mathbf{f}
$$

where $\mathbf{u}$ and $\mathbf{f}$ denote the coefficient vectors, $D$ and $A$ represent tridiagonal matrices determined by (12). For example, the subsystem for odd $n$ looks like this:

$$
\begin{gathered}
\left(\begin{array}{ccccc}
b_{1, m} & c_{1} & & & \\
a_{3} & b_{3, m} & c_{3} & & \\
& \ddots & \ddots & \ddots & \\
& & a_{J-3} & b_{J-3, m} & c_{J-3} \\
a_{J-1} & b_{J-1, m}
\end{array}\right)\left(\begin{array}{c}
u_{1, m} \\
u_{3, m} \\
\vdots \\
u_{J-3, m} \\
u_{J-1, m}
\end{array}\right)= \\
\left(\begin{array}{ccccc}
2 & -1 & & \\
-1 & 2 & -1 & \\
& \ddots & \ddots & \ddots & \\
& & -1 & 2 & -1 \\
& & & -1 & 2
\end{array}\right)\left(\begin{array}{c}
f_{1, m} \\
f_{3, m} \\
\vdots \\
f_{J-3, m} \\
f_{J-1, m}
\end{array}\right)
\end{gathered}
$$

The procedure to get the solution $u(\theta, \phi)$ to (8) is as follows: First, we get $f_{n, m}$, the spectral components of $f(\theta, \phi)$ by double Fourier series expansion. Then the right hand side of (13) is calculated to obtain the column vector $\mathbf{f}_{1}=A \mathbf{f}$. Finally, the tridiagonal matrix equation $D \mathbf{u}=\mathbf{f}_{1}$ is solved and $u(\theta, \phi)$ is obtained by inverse transform of $u_{n, m}$. Notice that the Poisson equation $\nabla^{2} u=f$ is just a special case of $\nabla^{2} u-\epsilon u=f$. Simply Setting $\epsilon=0$ in the above algorithm will give the solution to Poisson equation. Other simple elliptic equations, such as biharmonic equations can also be solved by this spectral method.

\subsection{Complexity Analysis}

Let us consider an elliptic equation with a grid size of $N \times N$ on unit sphere. If FEM were used, there would be a total of $N^{2}$ variables with matrix size $N^{2} \times N^{2}$. A crude Gauss elimination method will require $O\left(N^{6}\right)$ operations and the Gauss-Siedel relaxation will require $O\left(N^{4}\right)$ operations to converge. If the algorithms can take advantage of the fact that the matrix is sparse, it may reduce the number of operations to $O\left(N^{3}\right)$. However the computational complexity of the spectral method described above is only $O\left(N^{2} \log N\right)$.

\section{EXPERIMENTAL RESULTS}

We now present the results of applying the spectral method to solve the elliptic equations involved in the problems of surface reconstruction and $3 \mathrm{D}$ active contours.

\subsection{Surface Reconstruction}

In this problem, we apply the algorithm to some synthesized segmentation data to show how to choose the regularization parameter $\epsilon$ for different noise levels and for different shapes. The object center is assumed to be known or has been estimated in advance.

In the first experiment, we investigate the optimization of $\epsilon$ for different shapes. The reconstructions of a sphere and an ellipsoid are compared to illustrate the role of $\epsilon$. The Gaussian segmentation noise was introduced and the noise level in each sample direction is the same. In Fig. 1 and Fig. 2, $x$-axis represents the value of $\epsilon$ and $y$-axis represents standard deviation of the noise in the reconstructed surface. Fig. 1(a) shows that for a spherical shape which only contains low spatial frequency, $\epsilon$ should be as small as possible in order to filter out segmentation noise, while Fig. 1(b) shows that for an ellipsoidal shape which contains higher spatial frequencies, $\epsilon$ can be optimized to control the tradeoff between denoising and matching high spatial frequencies.

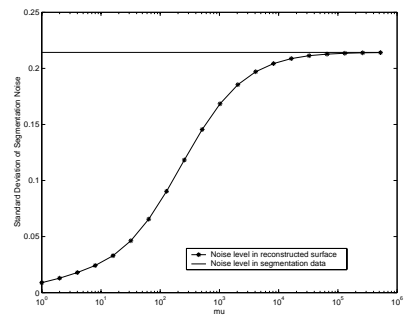

(a) Sphere

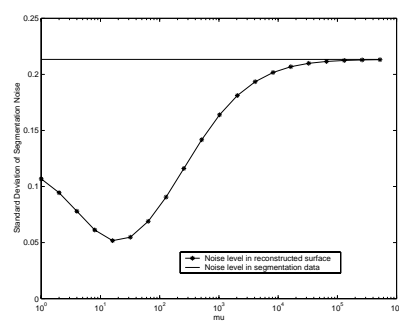

(b) Ellipsoid
Fig. 1. Noise in reconstructed surface vs. $\epsilon$ for different shapes

In the second experiment, the choice of $\epsilon$ for different segmentation noise levels was investigated. Different levels of Gaussian noise were added to the ellipsoidal shape. Fig. 2 shows that $\epsilon$ should be smaller for low $S N R$ segmentation data than for high $S N R$ segmentation data, which is as expected.

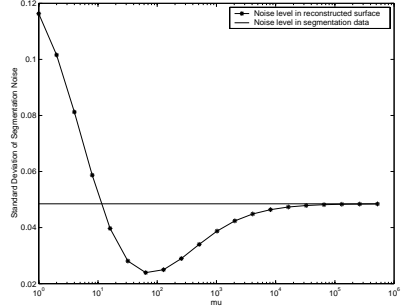

(a) $\sigma=0.05$

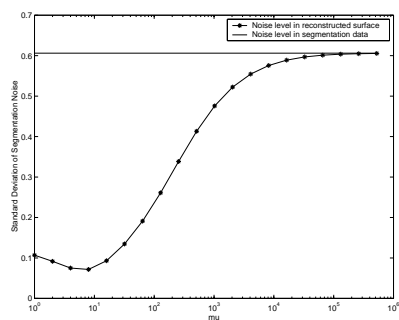

(b) $\sigma=0.60$
Fig. 2. Noise in reconstructed surface vs. $\epsilon$ for different noise levels

Surface reconstructions were accomplished in one iteration by the spectral method, while a single-grid relaxation algorithm may need more than 100 iterations.

\subsection{D Active Contours}

In this problem, the elliptic equations have to be solved in every evolution iteration. The advantage of using spectral method here 
is thus multiplied as compared to the surface reconstruction problem. We use double Fourier series to expand the radial descriptor of the 3D contour. Seven slices of an X-ray CT image were used to segment the liver. The set of edge maps for these CT slices is the input to our $3 \mathrm{D}$ active contour method. One of the CT slices is shown in Fig. 3(a) and its edge map is shown in Fig. 3(b). As in the surface reconstruction problem, the center of liver was estimated in advance. The contour was initialized as a sphere inside the liver. In every iteration, the position of the nearest edge point to a given point on the active contour was determined from the set of edge maps. The elliptic equation is then solved to determine the new position of the active contour. The role of $\alpha$ is inverse to the role of $\epsilon$, so it was set to 0.01 to keep the high spatial frequencies contained in the shape of liver. Fig. 3(c) and Fig. 3(d) show the contour's position in that particular slice after one and three iterations. In fact, the contour is very close to its convergent limit after only three iterations. Due to the fact that double Fourier series were truncated, the contour can not catch the sharp corner in the image. This is illustrated in Fig. 3(d).

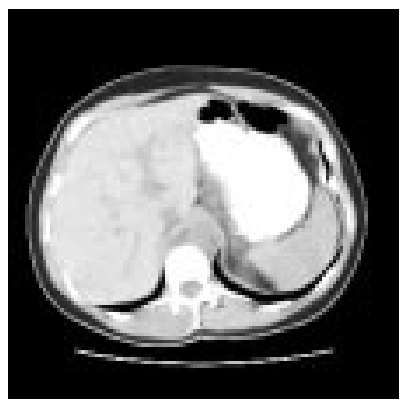

(a) a slice of CT image

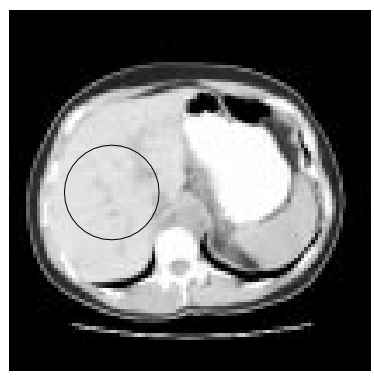

(c) iter $=1$

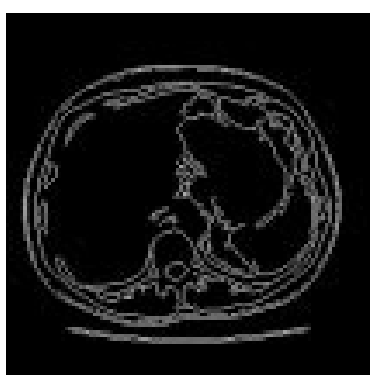

(b) Edge map

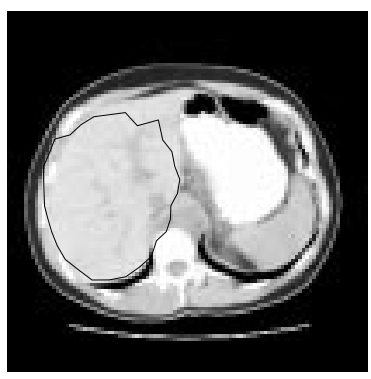

(d) iter $=3$
Fig. 3. Segmentation of liver in CT image by 3D active contours

\section{CONCLUSIONS}

In this paper, we have discussed the formulation of surface reconstruction and 3D active contours in the context of variational principles. It is shown that all these problems lead to solve elliptic equations on the unit sphere. A spectral method using double Fourier series as orthogonal basis functions has been applied to solve the elliptic equations. Compared to the complexity of $O\left(N^{4}\right)$ for iterative methods, the complexity of $O\left(N^{2} \log N\right)$ for spectral method is much lower. Some experimental results for surface reconstruction and 3D active contours were presented to illustrate the algorithms. In the experiment of $3 \mathrm{D}$ active contours, the edge map extracted by local edge detector is the only input to our algorithm. To improve the segmentation result, we propose to incorporate the intensity information obtained from image $I$ into the segmentation procedure as our future research. The optimization of the regularization parameter $\epsilon$ is also worth of further study.

\section{REFERENCES}

[1] B. Horn and M. Brooks, Shape From Shading, MIT Press, Cambridge, MA, 1989.

[2] D. Terzopoulos, "Image analysis using multigrid relaxation methods," IEEE Transactions on Pattern Analysis and Machine Intelligence, vol. 8, pp. 129-139, March 1986.

[3] L. D. Cohen and I. Cohen, "Finite-element methods for active contour models and balloons for 2-D and 3-D images," IEEE Transactions on Pattern Analysis and Machine Intelligence, vol. 15, no. 11, pp. 1131-1147, 1993.

[4] T. Simchony, R. Chellappa, and M. Shao, "Direct analytical methods for solving Poisson equations in computer vision problems," IEEE Transactions on Pattern Analysis and Machine Intelligence, vol. 12, no. 5, pp. 435-446, May 1990.

[5] H. Cheong, "Double Fourier series on a sphere: Applications to elliptic and vorticity equations," Journal of Computational Physics, vol. 157, no. 1, pp. 327-349, January 2000.

[6] S. Y. K. Yee, "Solution of Poisson's equation on a sphere by truncated double Fourier series," Monthly Weather Review, vol. 109, pp. 501-505, 1981.

[7] R. Courant and D. Hilber, Methods of Mathematical Physics, Interscience, 1953.

[8] J. P. Boyd, Chebyshev and Fourier Spectral Methods, Springer-Verlag, New York, 1989. 